\title{
The prevalence of adaptive immunity to COVID-19 and reinfection after recovery - a comprehensive systematic review and meta-analysis of 12011
}

\section{7 individuals.}

Rafal Al-Shebly', Rana Shalaby', Joshua T. Matizanadzo², Omran A. H. Musa'1, George Hindy', Luis Furuya-Kanamori', Nazmul Islam4, Mohammed Habibullah', Talal Al-Marwani', Rizeq F Hourani', Ahmed D Nawaz', Mohammad Z Haider', Mohamed M.

Emara, ${ }^{5,6}$, Farhan Cyprian', Suhail A. R. Doi' ${ }^{1}$, Tawanda Chivese

1. Department of Population Medicine, College of Medicine, QU Health, Qatar University, Doha, Qatar

2. Department of Public Health and Primary Care, Brighton and Sussex Medical School, United Kingdom

3. UQ Centre for Clinical Research, The University of Queensland, Herston, Australia

4. Department of Public Health, QU Health, Qatar University, Doha, Qatar

5. Basic Medical Sciences Department, College of Medicine, QU Health, Qatar University, Doha, Qatar

6. Biomedical and Pharmaceutical Research Unit, QU Health, Qatar University, Doha, Qatar.

\section{Objectives}

- Estimate the prevalence and longevity of detectable SARS-CoV-2 specific IgM, IgG, IgA antibodies, T and B memory cells during infection with SARS-CoV-2 and after recovery.

- Estimate the prevalence of COVID-19 reinfection.

- Evaluate the preventive efficacy of previous infection with SARS-CoV-2 against reinfection.

\section{Introduction}

- A key question that remains unanswered is whether infection with COVID-19 confers immunity and how long that immunity lasts.

- Several studies have shown that individuals infected with SARS-CoV-2 develop neutralizing antibodies to SARS-Cov-2 $(1,2)$, and that, up to 8 months later, most individuals who recover from COVID-19 have evidence of immunological memory (3-

7). However, many of these studies involve small numbers of participants and suffer from loss to follow up.

- Measuring the proportions of individuals with evidence of immunological memory of SARS-CoV-2 gives a relatively good idea of immunity against the virus after recovery. However, the best way of measuring immunity to COVID-19 after recovery is by measuring reinfection.

Methods

- This systematic review and meta-analysis followed the Preferred Reporting Items for

Systematic reviews and Meta-Analyses (PRISMA) guidelines (8).

- A synthesis of existing research was conducted.

- The Cochrane Library for COVID-19 resources, the China Academic Journals Full Text Database, PubMed, and Scopus were searched for studies conducted between 1 January 2020 to 1 April 2021.

- Studies with the relevant outcomes of interest, which compared COVID-19 infection

between individuals with and without prior infection were included.

- All included studies were assessed for quality and risk of bias.

- Pooled estimates of the prevalence of humoral and cellular immunity parameters and reinfection were obtained in a meta-analysis using bias adjusted synthesis methods.

- Proportions were synthesized with the Freeman-Tukey double arcsine transformation and binary outcomes using the odds ratio (OR).

- Heterogeneity between included studies was assessed using the I2 and Cochran's Q statistics and publication bias was assessed using Doi plots.

Fig.1 - Flow chart showing the search and inclusion of studies
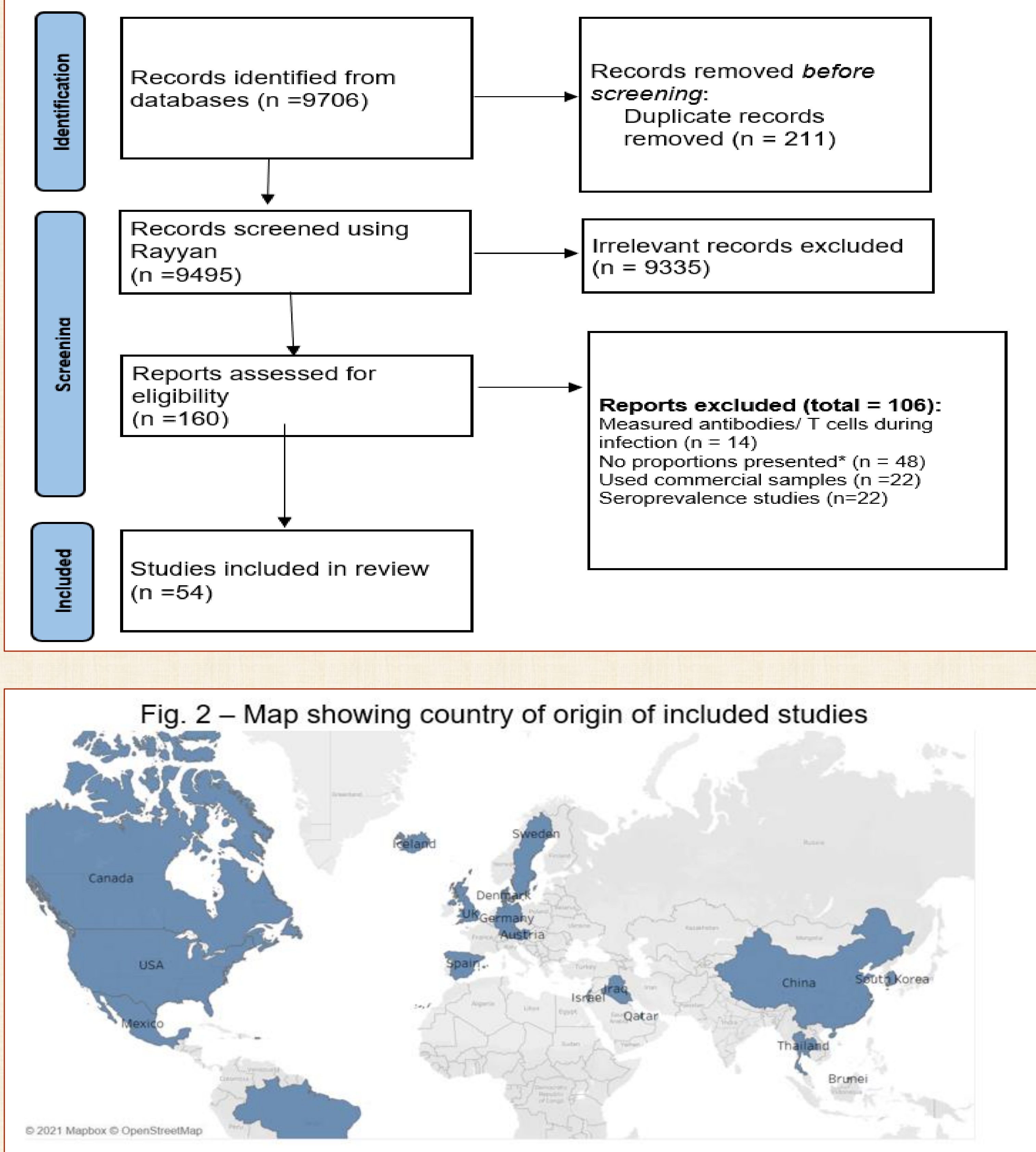

Results

A) Prevalence of SARS-CoV-2 specific IgG, IgM, CD4+ and CD8+ cells after recovery from COVID-19.

- The pooled prevalence of detectable IgG remained steadily elevated for months post recovery, where it was $89.0 \%$ within 1 month, $92.6 \%$ within $1-3$ months, $91.4 \%$ within 3-6 months and $90.4 \%$ after 6 months.

- The pooled prevalence of detectable IgM showed a downward trend with time, where it was 84.3\% within 1 month, 31.9\% within 1-3 months and around 51.6\%-61.4\% within 3-6 months.

- The prevalence of IgA was $63.4 \% 3$ months post recovery (9)

- The prevalence of detectable CD4+ T cells remained high even months post recovery; with levels being $100 \%$ within one month (6), $93.3 \%$ within $1-2$ months (11), $78.8 \%$ within 4.5 months (7) and $91.7 \%$ at $6-8$ months $(10)$

- $\mathrm{CD} 8+\mathrm{T}$ cells levels declined steadily from $70 \%$ at one month (10) to $50 \%$ at 6-8 months post recovery (6).

- The prevalence of memory B cells was $92.9 \%$ at 2-3 months post recovery (6) and $80.6 \%$ at $4-5$ months post recovery (12).

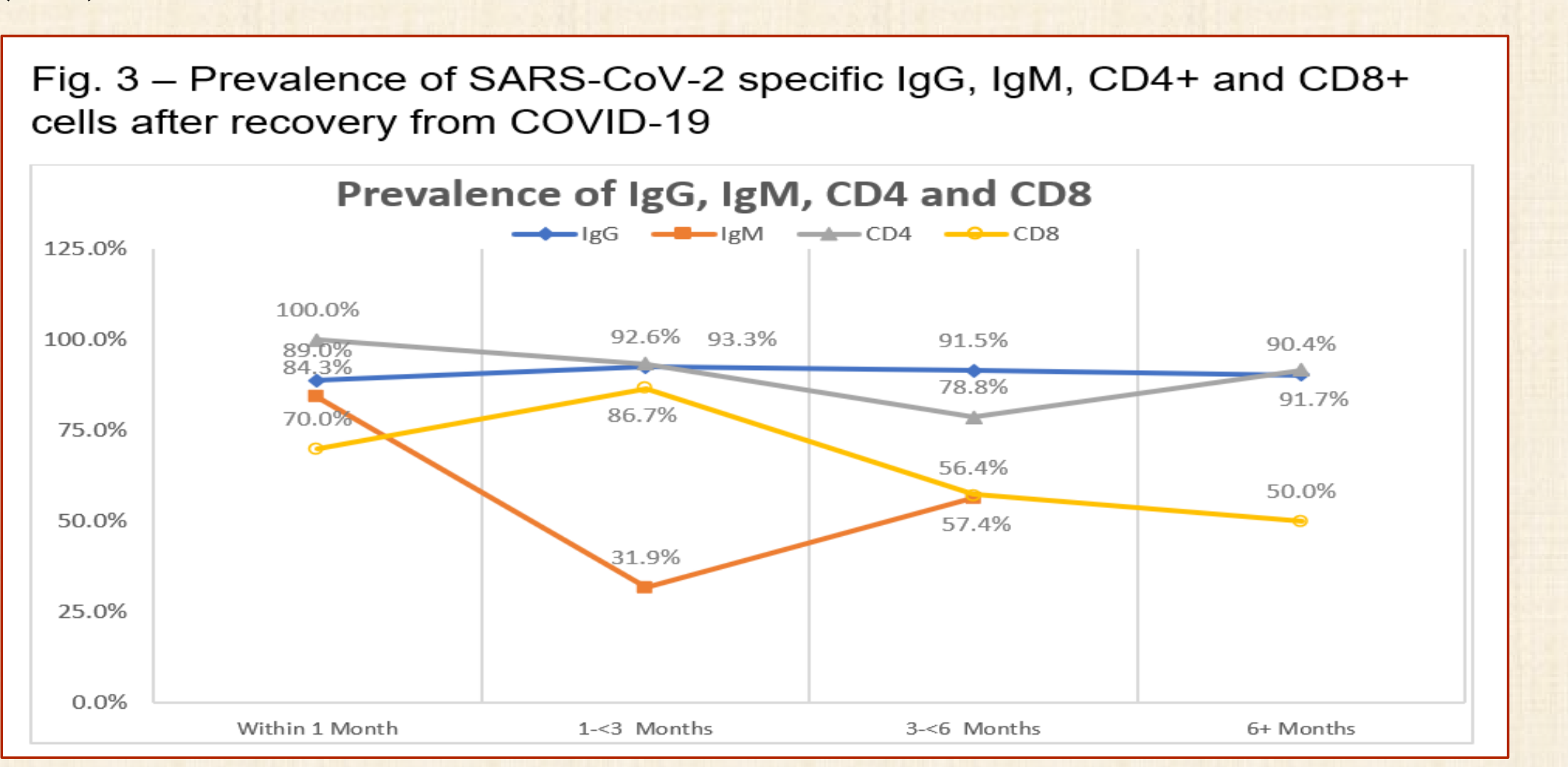

B) Repositivity and reinfection after recovery from COVID-19.

- The pooled prevalence of repositivity within one month was $2.0 \%$, whereas the pooled prevalence of repositivity at $2-3$ months after recovery was $0.2 \%$.

- The pooled prevalence of reinfection $\geq 3$ after months recovery from SARS-CoV-2 was $0.2 \%$.
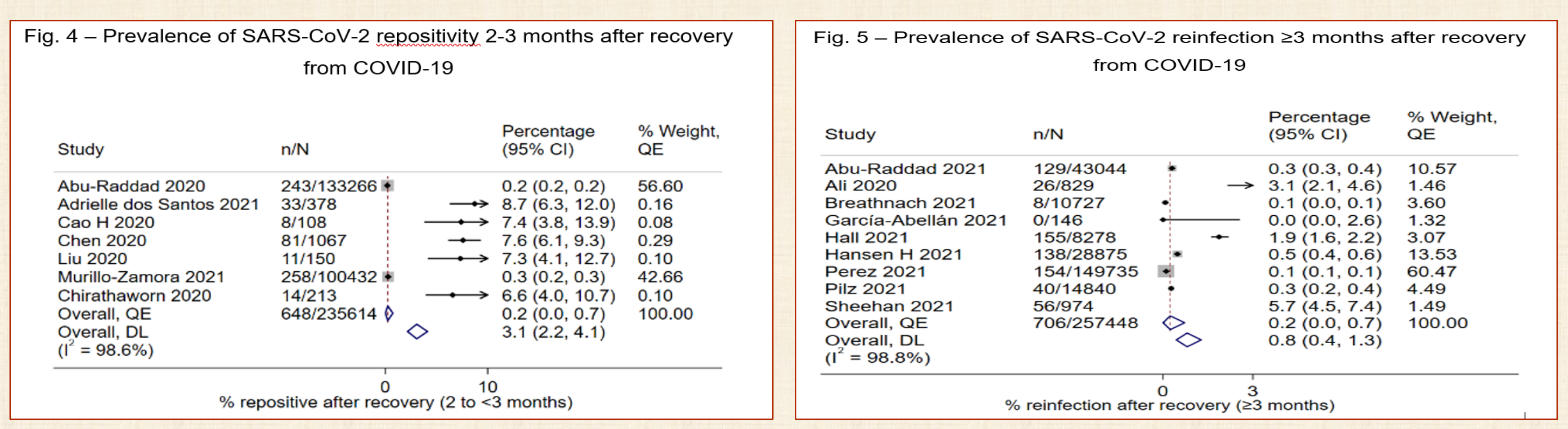

- Individuals previously infected with SARS-CoV-2 had an $81 \%$ reduction in odds of a reinfection

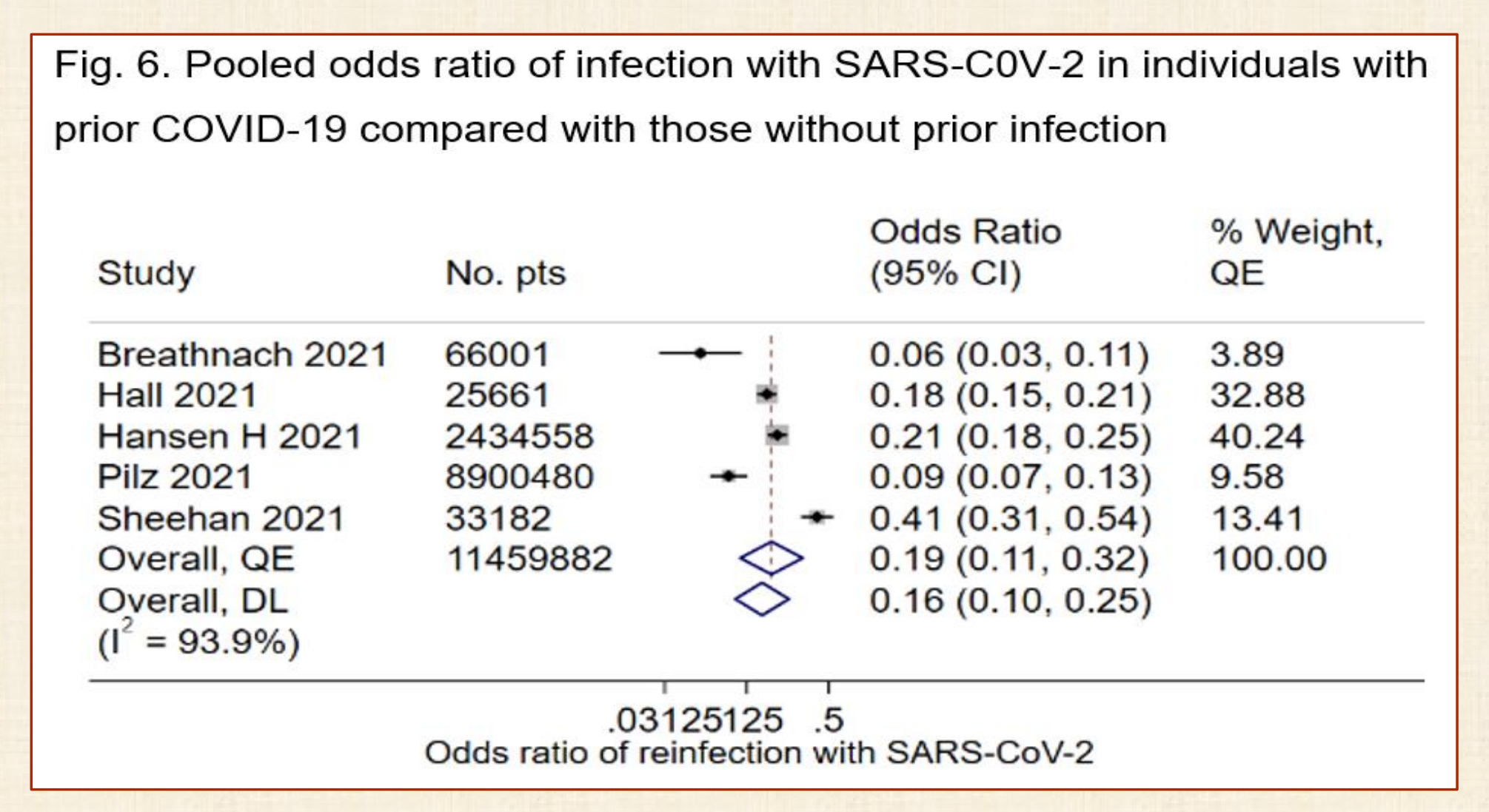

Conclusion

- Around $90 \%$ of people previously infected with SARS-CoV-2 had evidence of immunological memory to SARS-CoV-2, which was sustained for at least 8 months after recovery, and seemed to have a low risk of reinfection.

References

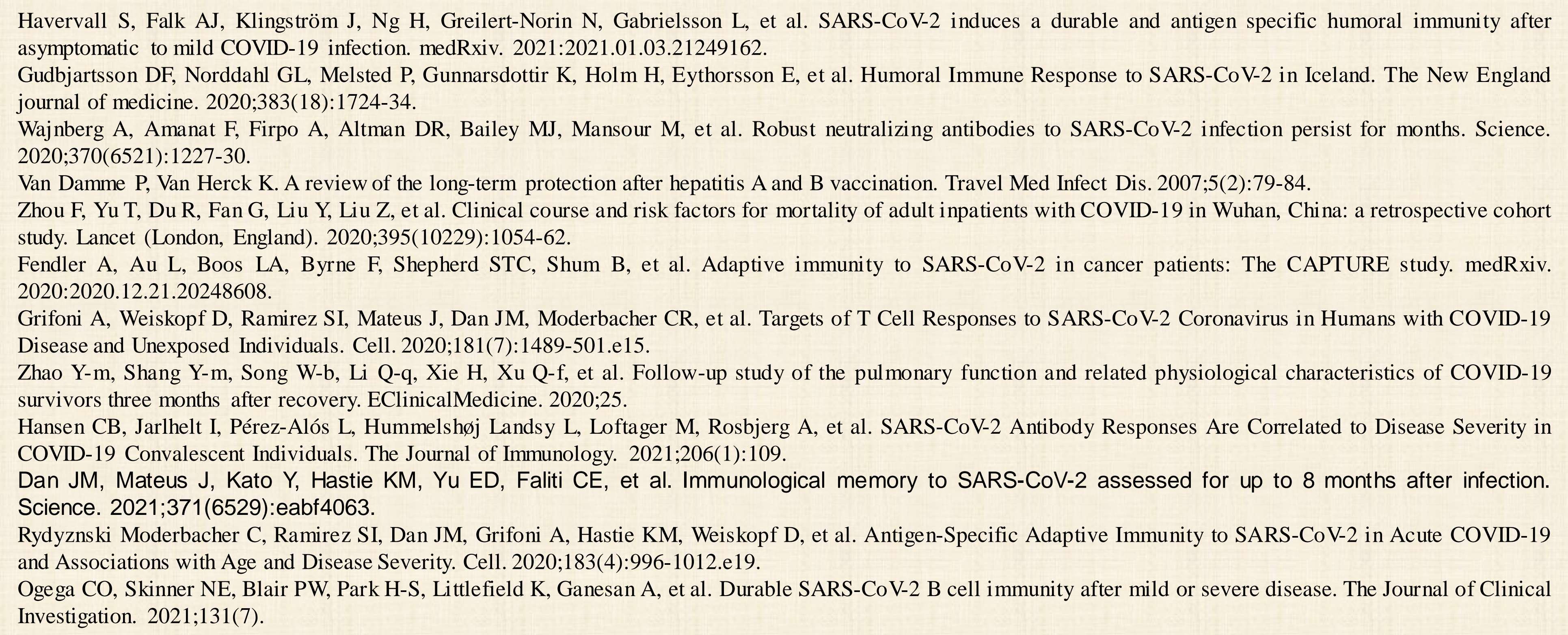

